\title{
Una gramática escolar interlingüuística. Hacia una enseñanza reflexiva de las lenguas en contextos multilingüüs
}

Hizkuntza arteko eskola-gramatika. Hizkuntzen irakaskuntza gogoetatsua testuinguru eleaniztunetan

An interlinguistic school grammar. Towards a reflective teaching of languages in multilingual contexts

\author{
Carmen Rodríguez-Gonzalo \\ Universitat de València-GIEL \\ crodrig@uv.es \\ https://orcid.org/0000-0002-8013-7503
}

Recibido / Noiz jaso den: 18/06/2021

Aceptado / Noiz onartu den: 23/10/2021

\begin{abstract}
Resumen
El reto de la educación plurilingüe requiere una enseñanza de las lenguas curriculares que promueva la reflexión sobre el uso y la transferencia lingüística para desarrollar la competencia comunicativa del alumnado. En este sentido, el proyecto Egramint se plantea la elaboración de una gramática escolar interlingüística como instrumento de formación docente y de intervención en el aula. Metodológicamente, es una investigación de carácter cualitativo-etnográfico en tres fases. En la primera, sobre la que versa este artículo, abordamos el análisis documental de los currículos oficiales del ámbito español para delimitar cómo se configura en ellos la gramática escolar. Los resultados muestran el predominio de la identificación sobre la manipulación o el uso reflexivo, así como los vínculos de la reflexión gramatical con la comprensión y elaboración de textos, en aspectos como la planificación y la revisión. Se aboga asimismo por un tratamiento interlingüístico, especialmente, en territorios con dos lenguas primeras.
\end{abstract}

\section{Palabras clave}

Formación del profesorado; currículos; reflexión sobre la lengua; competencia metalingüística; gramática escolar interlingüística.

\section{Sumario}

1. INTRODUCCIÓN. 2. LA GRAMÁTICA EN LOS CURRÍ́CULOS OFICIALES. 3. CONCLUSIONES. 


\begin{abstract}
Laburpena. Hezkuntza eleaniztunaren erronkak curriculum hizkuntzen irakaskuntza eskatzen du, ikasleen komunikazio gaitasuna garatzeko hizkuntzaren erabilera eta transferentziari buruzko hausnarketa sustatuko duena. Alde horretatik, hizkuntza arteko eskola-gramatika lantzea proposatzen du Egramint proiektuak irakaskuntza prestakuntzarako eta ikasgelan esku hartzeko. Metodologikoki, ikerketa kualitatibo-etnografiko bat da, hiru fasekoa. Lehenengo fasean, zeinari buruzkoa baita artikulu hau, Espainiako curriculum ofizialen azterketa dokumentala egingo dugu, eskola-gramatika curriculum horietan nola eratzen den zehazteko. Emaitzek erakusten dute identifikazioa dela nagusi manipulazioaren edo erabilera gogoetatsuaren gainean, bai eta gogoeta gramatikalak testuen ulermenarekin eta elaborazioarekin dituen loturak ere, planifikazioan eta berrikuspenean, besteak beste. Hizkuntzen arteko tratamendu baten alde egiten da, bereziki bi lehen hizkuntza dituzten lurraldeetan.
\end{abstract}

Gako hitzak. Irakasleen prestakuntza; curriculumak; hizkuntzari buruzko gogoeta; gaitasun metalinguistikoa; hizkuntzen arteko eskola gramatika.

\begin{abstract}
The challenge of multilingual education requires the teaching of curricular languages, which promotes reflection on the use and transfer of language to develop students' communicative competence. In this line of thinking, the Egramint project proposes the development of an interlinguistic school grammar as an instrument of teacher training and intervention in the classroom. Methodologically, it is a qualitative-ethnographic investigation in three phases. In the first phase, on which this article focus, we address the documentary analysis of the official curricula of the Spanish scope to delimit how school grammar is configured in them. The results show the predominance of identification over the manipulation or reflective use, as well as the links of the grammatical reflection with the comprehension and elaboration of texts, in aspects such as planning and revision. Interlinguistic treatment is also advocated, especially in territories with two first languages.
\end{abstract}

Keywords. Teacher training; curricula; reflection on the language; metalinguistic competence; interlinguistic school grammar.

\section{Introducción}

Los retos de una educación plurilingüue pasan por responder a la necesidad de los ciudadanos de utilizar las lenguas (del territorio y extranjeras) para usos progresivamente complejos, que implican el desarrollo del pensamiento y el dominio de procedimientos lingüuísticos diversos. Para atender esta necesidad social, la escuela ha de promover un uso reflexivo de las lenguas curriculares, que necesita de conocimientos gramaticales que puedan ser utilizados consciente y autónomamente por los escolares y de procedimientos metodológicos que permitan a los docentes de primaria y secundaria enseñar estos conocimientos en el aula (Locke, 2010; Rodríguez Gonzalo, 2012; Fontich y Camps, 2014; Ellis, 2016). Este es el reto que abordamos en el proyecto EGRAMINT ${ }^{1}$, que persigue la elaboración de una gramática escolar interlingüuística como instrumento de formación docente y de intervención en aula. La hipótesis de partida es que disponer de este tipo de gramática escolar facilitará alcanzar los objetivos de la educación plurilingüue que demanda la sociedad.

1 PID2019-105298RB-I00, Ministerio de Ciencia e Innovación-Aǵencia Estatal de Investigaación. 
Como es sabido, una de las prioridades del sistema educativo es el desarrollo de la competencia comunicativa de los estudiantes, considerada como competencia básica. Hoy en día, en consonancia con la sociedad en que vivimos, este desarrollo ha de ser plurilingüue, se ha de realizar en las diversas lenguas curriculares (del territorio y extranjeras) y ha de propiciar la autonomía del aprendiz, que habrá de abordar necesidades de comunicación diversas y proǵresivamente complejas. Ello requiere un cierto grado de reflexión sobre las lenguas que se utilizan, como se ha puesto de relieve tanto desde lenguas primeras (Nadeau y Fisher, 2011; Camps, 2014, 2017) como desde lenguas extranjeras (Ellis, 2016; Nassaji, 2017). Se trata, por tanto, de que desde la educación lingüuística se contemple como necesario el desarrollo de la competencia metalingüústica, transversal a las distintas vertientes de la competencia comunicativa (praǵmática, lingüuística, sociolingüústica y literaria). Para este desarrollo, los conocimientos gramaticales son imprescindibles.

Actualmente, estamos de nuevo ante la perspectiva de un cambio curricular (MEFP, 2020). Por ello, reflexionar sobre lo que nos dicen los currículos vigentes y sobre el momento en el que nos encontramos puede ser una buena manera de pensar desde dónde partimos y cuál es el camino que tenemos por delante.

Los currículos educativos desarrollados a partir de la LOMCE (2013) recogen la necesidad de reflexión sobre los mecanismos lingüuísticos que regulan la comunicación y señalan que estos conocimientos no han de entenderse como un fin en sí mismos, sino que han de servir de base para el uso correcto de la lengua. Así, tanto en primaria como en secundaria, se declara en las introducciones a los currículos de Lengua castellana y Literatura²

La finalidad de la reflexión lingüústica es el conocimiento progresivo de la propia lengua, que se produce cuando el alumno percibe el uso de diferentes formas lingüísticas para diversas funciones, y cuando analiza sus propias producciones y las de los que le rodean para comprenderlas, evaluarlas y, en su caso, corregirlas. [...] El bloque 4, Conocimiento de la Lengua, responde a la necesidad de reflexión sobre los mecanismos lingüústicos que regulan la comunicación, y se aleja de la pretensión de utilizar los conocimientos linguuuísticos como un fin en sí mismos para devolverles su funcionalidad original: servir de base para el uso correcto de la lengua.

Este planteamiento arranca ya desde los años 90 del siǵlo pasado, cuando los currículos desarrollados a partir de la LOGSE (1990) plantearon la necesidad de

2 La cita es idéntica en los anexos de Lengua castellana y Literatura de primaria y secundaria de la legislación estatal (RD 126/2014 de 28 de febrero, por el que se establece el currículo básico de la Educación Primaria y RD 1105/2014 de 26 de diciembre, por el que se establece el currículo básico de la Educación Secundaria Obligatoria y el Bachillerato). 
repensar la enseñanza de la gramática, centrada entonces en el aprendizaje de mecanismos formales desvinculados de la conciencia que los hablantes tienen del uso de la lengua, con gran presencia de nociones estructuralistas y de gramática generativa. Contra este planteamiento ya se alzaban numerosas voces, como la de Landero (1999):

Tengo un joven amigo que, después de diez años de estudiar gramática, se ha convertido al fin en un analfabeto de lo más ilustrado. Se trata de un estudiante de bachillerato de nivel medio, como tantos otros, y aunque tiene dificultades casi insalvables para leer con soltura y criterio el editorial de un periódico, es capaz sin embargo de analizar sintácticamente el texto que apenas logra descifrar. Su léxico culto es pobre, easi de supervivencia, pero eso no le impide despiezar morfológicamente, como un buen técnico que es, las palabras cuyo significado ignora y enumerar luego de corrido los rasgos del lenguaje periodístico, y comentar las perífrasis verbales y explayarse aún en otras lindezas formales de ese estilo. [...] Uno no tiene nada contra la gramática, pero sí contra la intoxicación gramatical que están sufriendo nuestros jóvenes. Uno está convencido de que, fuera de alǵunos rudimentos teóricos, la gramática se aprende leyendo y escribiendo, y de que quien llegue, por ejemplo, a leer bien una página, entonando bien las oraciones y desentrañando con la voz el contenido y la música del idioma, ese sabe sintaxis. Solo entonces, como una confirmación y un enriquecimiento de lo que básicamente ya se sabe, alcanzará la teoría a tener un sentido y a mejorar la competencia lingüústica del usuario.

De la necesidad de vincular uso y reflexión, se han derivado dos interpretaciones en el ámbito educativo: a) en la primera, solo interesan los conocimientos lingüuísticos que tienen que ver con la normativa de uso de las lenǵuas (aspectos normativos de la ǵramática, ortografía y puntuación, en el caso de la lenǵua escrita); b) para la segunda, el dominio de los usos de la lengua requiere cierta capacidad de control, que necesita de conocimientos gramaticales explícitos.

En el primer caso, el aprendizaje de la lengua se ha entendido exclusivamente como el aprendizaje de las normas de un código, establecidas desde instancias ajenas al hablante, que determinan lo que es correcto o incorrecto o lo que debe o no decirse en un contexto dado. Es innegable la responsabilidad de la escuela en relación con el dominio de la normativa, pero el uso de la lengua no se puede reducir a este tipo de conocimiento (Mantecón Ramírez, 1989; Rodríguez-Gonzalo, 2012).

La segunda interpretación ha explorado las vías de acercamiento entre uso y reflexión en el aula, partiendo de la consideración de que la reflexión del hablante (en cualquier tramo de edad) desempeña un papel fundamental en su conocimiento de las lenguas que utiliza y en la progresiva formación de su voz de autor, especialmente, en los usos escritos. Las lenguas se configuran por el uso que se hace de ellas y conocer todas las posibilidades que ofrecen ayuda a tomar decisiones sobre cómo decir algo y también a valorar los efectos (de significado y de 
interpretación) de esta toma de decisiones. Para que la reflexión se pueda considerar como tal, es necesario que se asiente en conocimientos que se manifiestan con distinto grado de explicitud (Camps, 2000; Zayas, 2011a).

Desde esta perspectiva, se entiende que, para elaborar un texto adecuado a una situación discursiva determinada, sea oral, escrita o multimodal, se requiere de una intensa actividad metalingüuística, que solo es posible si se conocen los elementos que la lengua pone a disposición de los hablantes y si se ha aprendido a utilizarlos conscientemente, lo que dista mucho de un aprendizaje de normas y definiciones (Kolln y Gray, 2014; Durán y Rodríguez Gonzalo, 2019).

Estas dos vías coexisten en la actualidad en nuestro sistema educativo y en otros de nuestro entorno (Costa, 2020), a veces ignorándose y a veces con múltiples malentendidos. En los currículos oficiales analizados, estos contenidos, en el caso de las lenguas primeras, se agrupan en bloques con denominaciones como «Conocimiento de la lenǵua» o «Reflexión sobre la lengua y sus usos», que se asimilan con frecuencia a «conocimientos gramaticales». Estos bloques incluyen no solo contenidos morfosintácticos, sino también fonológicos, léxicos, semánticos y pragmáticos, además de los referidos a ortoǵrafía y puntuación, lo que nos lleva a plantearnos cuáles deben ser los contenidos de una gramática escolar. ¿Es lo mismo una gramática escolar que una gramática divulgativa? ¿Cuáles son las diferencias?

Veamos cómo se presentan dos importantes gramáticas divulgativas: la Nueva Gramática Básica de la Lengua española (NGBLE, 2011) y la Gramàtica básica i d'ús de la llengua catalana (GBU, 2019).

La NGBLE (2011) declara en su presentación:

Ahora sale a la luz la Nueva gramática básica de la lengua española. Esta versión se dirige expresamente al amplio espectro de hispanohablantes que, habiendo recibido una primera instrucción en sus estudios de primaria y de secundaria, deseen acercarse a comprender mejor el funcionamiento de la lengua. (p. XVII) $[\ldots]$ conjuga la dimensión descriptiva con la normativa $[\ldots]$ se centra en el español estándar y elige en cada caso las opciones cultas mayoritarias entre los hispanohablantes. Aspira, en definitiva, a convertirse en la gramática de todo el mundo. (p. XVIII).

Por su parte, la GBU (2019), también en la presentación, señala que

S'adreça al públic no especialitzat i molt específicamente al món de l'Administració, dels mitjans de comunicación i de l'ensenyament, com a obra de consulta que pot orientar en la interpretación i l'aplicació normativa de l'IEC. [...] está concebuda [...] com a manual de consulta adaptat a usuaris amb coneixements gramaticals generals que volen resoldre dubtes d'ús. (p. IX) [...] La GBU [...] es presenta com una gramática descriptiva i normativa: mostra els usos i, en cas que calgui prescripció, els valora i, si convé, els jerarquitza. Defuig, 
però l'aproximació basada en el criteri de correcció o incorrecció, i adopta el criteri d'adequació als diversos registres (formals, molt formals i informals), que té relació amb l'àmbit de comunicació, més o menys general. (p.XI) ${ }^{3}$.

En ambos casos, las gramáticas se destinan a un público general, con conocimientos gramaticales de base («habiendo recibido una primera instrucción en sus estudios de primaria y secundaria» NGBLE, «com a manual de consulta adaptat a usuaris amb coneixements gramaticals generals» GBU) y con vocación descriptiva y normativa. Es decir, como gramáticas divulgativas que son, ponen al alcance de los hablantes, de forma accesible si se tiene una base previa, conocimientos sobre la lengua y su uso. Esta base previa, que en ambos casos dan por supuesta, solo puede formarse en el ámbito escolar.

Nos acercamos, entonces, a qué entendemos por gramática escolar. Es una gramática en la que se han de construir estas nociones que las gramáticas divulgativas dan por sabidas y sobre las que se asientan. Y son nociones que guardan relación no solo con cómo es la lengua sino también con cómo se usa. En este sentido, la GBU (2019) aporta una observación de gran importancia. El uso se puede entender como acatamiento de la norma (correcto-incorrecto) o como adecuación a los distintos registros. En el primer caso, no cabe la reflexión, pero sí en el segundo. Si consideramos las variantes expresivas propias de los distintos registros, estaremos ante una gramática de elecciones o ante una gramática retórica (Kolln y Gray, 2014).

Sabemos, pues, que una gramática escolar ha de permitir que los alumnos vayan construyendo esas nociones básicas sobre la lengua y se vayan acercando a las posibilidades que ofrece el uso de estas. Y como este proceso no es espontáneo, es un aprendizaje que se ha de ir realizando en las aulas.

Aquí es donde situamos nuestro proyecto de elaboración de una gramática escolar interlingüústica (Egramint). Consideramos que, para que se pueda considerar una gramática escolar, ha de tener en cuenta el proceso de enseñanza y aprendizaje y, por ello, ha de integrar los contenidos y las formas de trabajo en el aula (el qué y el cómo). Ha de dar respuesta, asimismo, a las necesidades de los

3 Se diriǵe al público no especializado y muy específicamente al mundo de la administración, de los medios de comunicación y de la enseñanza, como obra de consulta que puede orientar en la interpretación y la aplicación normativa del Institut d'Estudis Catalans (IEC) [...] está concebida [...] como manual de consulta adaptado a usuarios con conocimientos gramaticales generales que quieren resolver dudas de uso (p. IX). La GBU [...] se presenta como una gramática descriptiva y normativa: muestra los usos y, si es necesario, la prescripción, los valora y, si conviene, los jerarquiza. Huye, sin embargoo, de la aproximación basada en el criterio de corrección o incorrección, y adopta el criterio de adecuación a los diversos registros (formales, muy formales e informales), lo que tiene relación con el ámbito de comunicación, más o menos general (p. XI). [La traducción es nuestra]. 
hablantes: saber utilizar diversas lenguas y saber aprovechar los conocimientos lingüústico-comunicativos en todas ellas. Hablamos entonces de desarrollar una competencia interlingüústica, que permita transferir contenidos comunes -básicamente los contenidos de carácter discursivo- y que posibilite el contraste de las especificidades de cada lengua, asentadas en conceptos gramaticales compartidos (Ruiz Bikandi, 2008; Guasch, 2011).

Abordar este planteamiento requiere formar a los docentes (de primaria y de secundaria) para que promuevan el uso reflexivo de las lenguas curriculares. Para ello, tan importante es seleccionar los conocimientos gramaticales necesarios para que puedan ser usados consciente y autónomamente por los estudiantes, como disponer de procedimientos metodológicos que permitan a los docentes trabajar reflexivamente en el aula.

Este es el contexto en que nos situamos. Pretendemos actualizar el concepto de gramática escolar de forma que sea un instrumento útil en manos de los docentes, para abordar la educación plurilingüüe de los estudiantes, tanto de primaria como de secundaria.

Nuestra investigación es de carácter cualitativo-etnográfico (Woods, 1996; Cambra, 2013), de tipo interaccionista (Ellis, Edwards y Smagorinsky, 2010) articulada en tres fases: a) una primera fase de análisis documental de los currículos oficiales vigentes, previa al diseño y pilotaje del dispositivo de intervención (a partir del modelo de secuencias didácticas de ǵramática, SDG, Camps, 2006); b) una segunda fase de implementación en aula para obtener evidencias empíricas sobre la enseñanza gramatical; c) una tercera fase de configuración de la gramática escolar interlingüuística, como instrumento de apoyo docente.

\section{La gramática escolar en los currículos oficiales}

Como hemos señalado, uno de los objetivos del proyecto es aproximarnos al concepto de gramática escolar que aparece en los currículos oficiales desarrollados a partir de la LOMCE (2013) para las distintas lenguas del sistema educativo español. Corresponde a la primera fase, que abordamos desde la perspectiva metodológica del análisis documental (Bowen, 2009).

No pretendemos aquí realizar un análisis crítico de estos documentos (v. Durán, 2010; Ruiz Bikandi, 2010; Bosque y Gallego, 2018), sino poner de manifiesto qué concepción de gramática escolar se presenta en ellos. Somos conscientes de que el llamado currículo prescrito adopta «macrodecisiones» (Costa, 2020) y que desde este grado de generalidad puede resultar sencillo hacer afirmaciones que no son fáciles de llevar a la práctica (Jover, 2008; Bosque y Gallego, 2018). Consideramos, no obstante, que resulta de interés conocer cómo se presentan los contenidos gramaticales y, especialmente, los aprendizajes que se esperan de los alumnos. 
El corpus de nuestro análisis está formado por todos los currículos oficiales vigentes $^{4}$ (el decreto de mínimos estatal y los autonómicos) de las distintas lenguas curriculares, tanto lenguas primeras de los distintos territorios (castellano, catalán, gallego, euskera, aragonés y asturiano) como extranjeras (primeras y segundas). En total, son 128 documentos, correspondientes a primaria y secundaria (v. fig. 1).

\section{PRIMARIA (62 documentos)}

\section{SECUNDARIA (ESO) (66 documentos)}

L1-Castellano: 19

L1-Catalán: 4

L1-Catalán: 4

Cataluña, C. Valenciana, I. Baleares, Aragón

Cataluña, C. Valenciana, I. Baleares, Aragón

L1-Euskera: 3

L1-Euskera: 3

Euskadi; Navarra (modelos A y D)

Euskadi; Navarra (modelos A y D)

L1-Gallego: 1

L1-Gallego: 1

L1-Lengua aragonesa: 1

L1-Lengua aragonesa: 1

L1-Lengua asturiana: 1

L1-Lengua asturiana: 1

LEX-1 (primera lengua extranjera): 22

LEX-2 (segunda lengua extranjera): 11
LEX-1 (primera lengua extranjera): 19

LEX-2 (segunda lengua extranjera): 18

Figura 1. Documentos curriculares. Corpus de análisis (elaboración propia).

En el análisis de estos documentos se han codificado todas las indicaciones sobre contenidos y procedimientos de enseñanza de lenguas relacionados con la reflexión metalinguuística, como macrocategoría, entendida como la reflexión del hablante sobre las lenguas y sus usos.

Esta codificación se ha realizado en dos fases: una primera, realizada entre todos los miembros del equipo ${ }^{5}$, que permitió el establecimiento de la categorización inicial, mediante un procedimiento interjueces, y una segunda, realizada mediante el programa de análisis de contenido Atlas.ti-9, que ha permitido sistematizar la primera categorización, a partir del rastreo de los términos clave acordados como identificadores de cada categoría.

Las dos grandes categorías en que desǵlosamos la reflexión metalingüuística inicialmente son «Conocimiento de la lengua» $\mathrm{y}$ «Uso de la lengua». En ambos casos, se subdividieron entre conocimientos declarativos (qué, objeto) y operacio-

4 El corpus se cerró en julio de 2021.

5 Formado por investigadoras de las siguientes universidades: UV, UJI, UAB, UVic-UCC, UCA y Unileón. 
nes cognitivas que implican formas de abordar estos conocimientos en el proceso de enseñanza y aprendizaje (cómo) y que aparecen formulados en los currículos en los apartados de contenidos, de criterios de evaluación o de estándares de aprendizaje, según los casos.

La categoría «Conocimiento de la lenǵua», en los ítems referidos a contenidos declarativos de los currículos, incluye los que tienen como referencia los contenidos gramaticales referidos a la palabra, a la oración y al texto (siguiendo en este último caso, los criterios establecidos en Cuenca, 2010, p.116), así como los contenidos semánticos y fonológicos relacionados con ellas.

En cuanto a la forma de abordar estos conocimientos, se tomó como referencia inicial la clasificación de operaciones cognitivas propuesta en la taxonomía de Bloom, en la versión revisada por Anderson y Kratwohl (2001), que establece seis categorías: recordar, comprender, aplicar, analizar, evaluar, crear. Esta clasificación se contrastó con los tipos de actividades gramaticales propuestos por Chartrand (2003) y Zayas (2011b). Chartrand (2003) establece siete tipos de actividad (motivar la reflexión metalingüuística; aprender a observar la lengua en su contexto de uso; descubrir una regla, una regularidad o un mecanismo de la lengua; construir procedimientos de aplicación de las normas; construir procedimientos de detección y corrección de errores; evaluación de los procesos y de las producciones lingüústicas de los alumnos y, por último, iniciación a la consulta de obras de referencia sobre la lengua, como gramáticas o diccionarios), relacionables con los cinco primeros tipos de la taxonomía de Bloom revisada (excepto «crear»). Zayas, por su parte, plantea diez tipos de actividades (clasificar, comparar, ejemplificar, juzgar, interpretar, completar, manipular, reescribir, integrar y componer un texto), que agrupa según las actividades cognitivas que implican, con claras relaciones con la taxonomía de Bloom-Anderson y Kratwohl.

Estas categorías, referidas a las operaciones cognitivas, se relacionan con el proceso de adquisición de los conceptos científicos (Barth, 2004), que se diferencia de la formación espontánea de conceptos, entendida como proceso espontáneo de reagrupación de los elementos de la realidad. La estructura operatoria de un concepto, según esta autora, necesita de denominación, identificación de atributos y atribución a ejemplos, para lo cual se requiere de procesos como la percepción siǵnificativa, la comparación, la realización de inferencias que permitan establecer conclusiones y verificarlas, para luego generalizar la experiencia y atribuirla a realidades semejantes.

6 «[... la gramática del texto se ocupa de los mecanismos formales, fundamentalmente gramaticales y léxicos, que hacen que un conjunto de oraciones formen una unidad superior desde un punto de vista semántico y comunicativo. [...] entendemos la gramática del texto como equivalente a la cohesión o sintaxis textual» (Cuenca, 2010, p. 11). 
Por su parte, en la categoría «Uso de la lengua», se consideraron conocimientos declarativos (qué), es decir, todos aquellos que el hablante ha de conocer explícitamente para poder usarlos. Así, las subcategorías establecidas fueron: aspectos normativos de la escritura (ortografía y puntuación) y de la oralidad (prosodia), variedad (de lenguas y de registros en cada lengua) y herramientas de consulta (TIC y gramáticas). Sobre el uso reflexivo de la lenǵua (cómo), se han tenido en cuenta las operaciones que implican reflexión y control del uso, tanto en escritura como en oralidad (por ejemplo, la planificación o el control de rasgos lingüuísticos de los géneros discursivos).

Por último, se aǵrupó en la categoría «Bases», todos aquellos aspectos referidos a objetivos y planteamientos metodológicos generales que apuntaban a la reflexión sobre las lenguas (reflexión gramatical, transferencia, plurilingüiismo, especialmente).

En la figura 2 se puede observar una esquematización de las categorías generales empleadas.

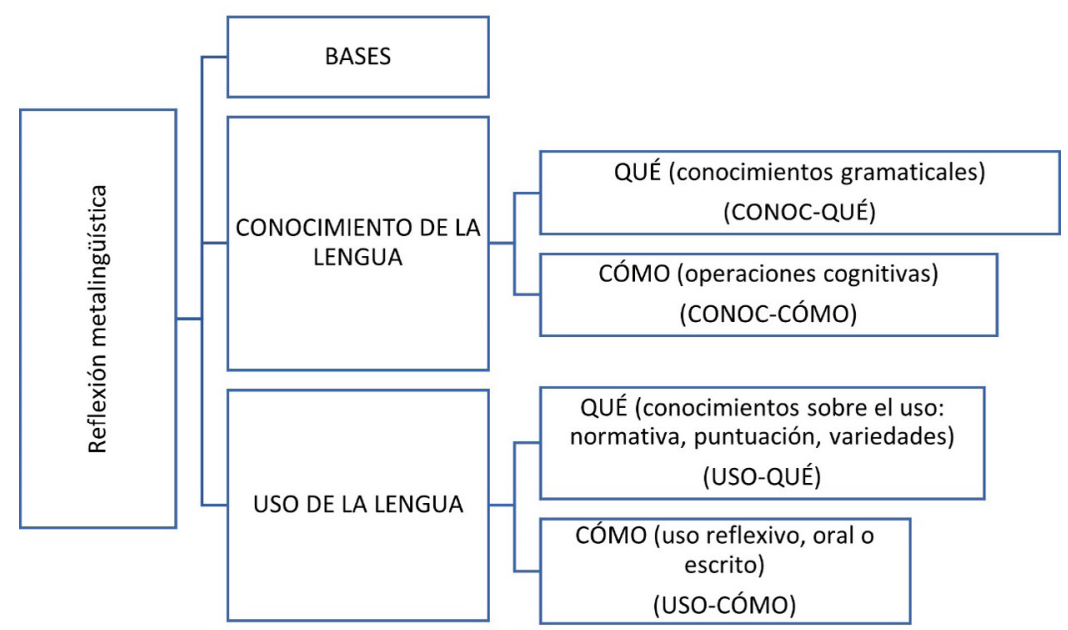

Figura 2. Categorías de análisis (elaboración propia).

En este artículo, en relación con el análisis, nos centramos únicamente en una parte del corpus, el relativo a las lenguas primeras (L1), y en los resultados iniciales obtenidos sobre las operaciones cognitivas relacionadas con los conocimientos gramaticales, que aparecen sintetizados en las figuras 3 y $4^{7}$. Estas

7 Los datos que se presentan corresponden a porcentajes sobre tablas código-documento normalizadas en Atlas.ti-9 y sin fusión de redundancias. La normalización hace equivalente la densidad de codificación de todos los documentos de la tabla, ya que se considera que cada documento tiene la misma cantidad de citas. 
operaciones están aǵrupadas en la categoría Conocimiento de la lenǵua-Cómo (CONOC-CÓMO) y han sido rastreadas en los documentos mediante distintas palabras clave (verbos y sustantivos deverbales, según si se formulan como contenidos, como criterios de evaluación o como estándares de aprendizaje). Los datos obtenidos, si bien de carácter orientativo, nos aproximan al planteamiento sobre la construcción de conceptos básicos y sobre el desarrollo de la competencia metalingüuística de los estudiantes que se observa en los documentos curriculares.

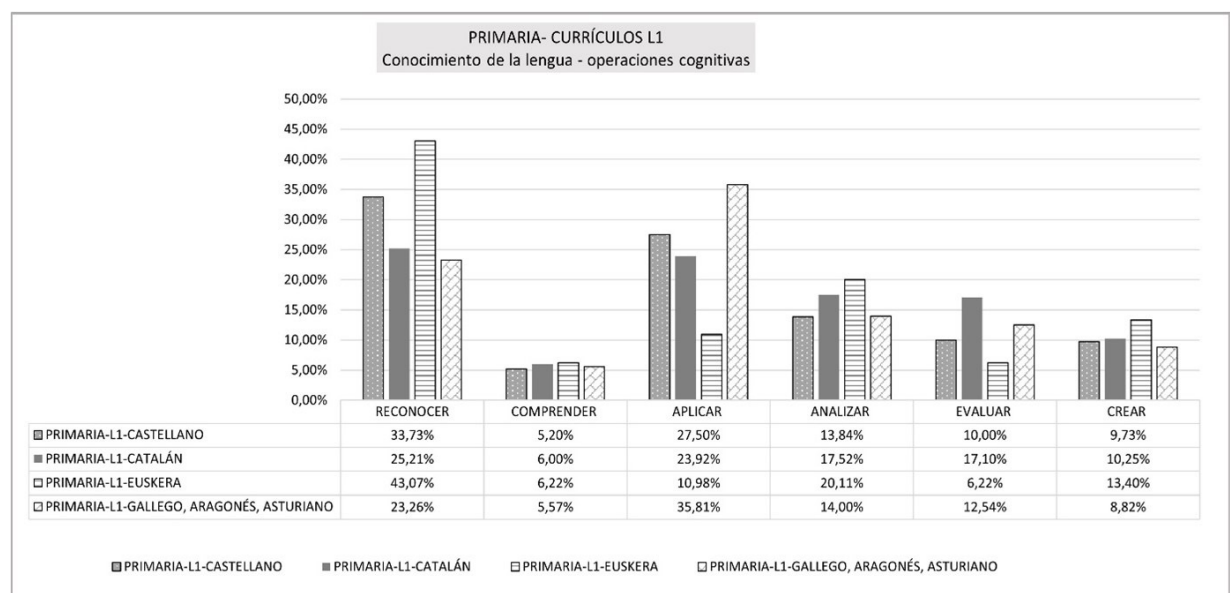

Figura 3. Operaciones cognitivas sobre el conocimiento de la lengua en los currículos de primaria L1 (elaboración propia)

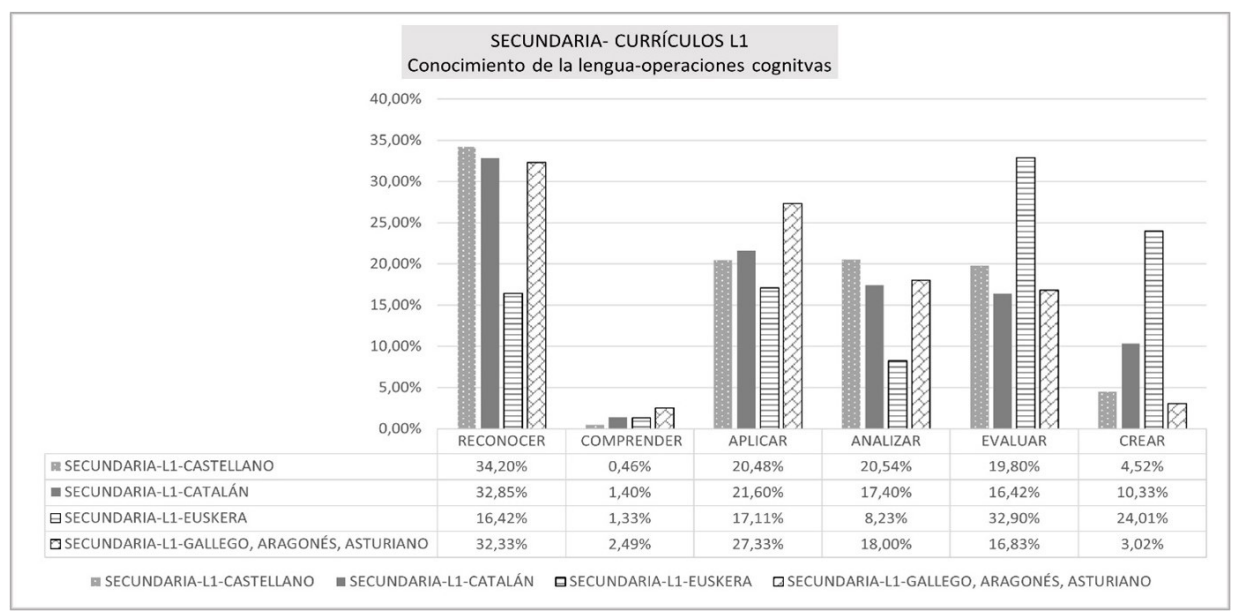

Figura 4. Operaciones cognitivas sobre el conocimiento de la lengua en los currículos de secundaria L1 (elaboración propia). 
La categoría «Reconocer» (CONOC-CÓMO-REG) incluye todas aquellas operaciones cognitivas que implican identificación o localización de los ítems gramaticales recogidos en la categoría CONOC-QUÉ. Según Bloom-Anderson y Krathwohl (2001), esta categoría incluye operaciones en las que se reconozea y se traiga a la memoria información relevante de la memoria de largo plazo. Barth (2004) considera la percepción significativa como el primer paso de la conceptualización.

Los datos de las figuras 3 y 4 muestran que es la categoría más presente tanto en primaria como en secundaria, con algunas precisiones. En la figura 5, recogemos el contraste de porcentajes.

\begin{tabular}{|lc|}
\hline Documentos curriculares & $\begin{array}{c}\text { Categoría: Reconocer } \\
\text { primaria - secundaria }\end{array}$ \\
\hline L1-Castellano & $33,73 \%-34,20 \%$ \\
\hline L1-Catalán & $25,21 \%-32,85 \%$ \\
\hline L1-Euskera & $43,07 \%-16,42 \%$ \\
\hline L1-Gallego, aragonés, asturiano & $23,26 \%-32,33 \%$ \\
\hline
\end{tabular}

Figura 5. Categoría: Reconocer. Presencia en currículos de L1, primaria-secundaria (elaboración propia).

En primaria, la identificación remite a los inicios de la conceptualización tanto en unidades básicas referidas a la aproximación al códiǵo escrito (sílabas, palabras) como a la identificación de las clases de palabras, grupos, funciones sintácticas y elementos de cohesión textual. Se observa una cierta progresión en los cursos, ya que la identificación se combina con elementos de reflexión (1csabiendo que..., 1e-explicación) o de uso (1d-entendido aquí como aplicación):

(1a) Reconoce y lee las vocales y las letras consonantes. (Primaria-1. ${ }^{\circ}$ Castilla-León-L1).

(1b) Identificar el sujeto y predicado en diferentes oraciones. (Primaria-2. ${ }^{\circ}$ Castilla-León-L1).

(1c) Localizar la sílaba tónica sabiendo que puede ocupar diferentes lugares en las palabras. (Primaria-4. ${ }^{\circ}$ Castilla-León-L1).

(1d) Reconocimiento y uso de algunos conectores textuales (de orden, contraste y explicación) y de los principales mecanismos de referencia interna, tanto gramaticales (sustituciones pronominales) como léxicos (elipsis y sustituciones mediante sinónimos e hiperónimos). (Primaria-5. Aragón-L1).

(1e) Reconocimiento de las distintas clases de palabras y explicación reflexiva de su uso en situaciones concretas de comunicación (nombre, verbo, adjetivo, preposición, adverbio, conjunción, pronombres, artículos, interjecciones). (Primaria-6. ${ }^{\circ}$ Aragón-L1). 
En secundaria, la identificación aparece en los primeros cursos de la etapa combinada con el análisis (1f, 1g) y, en ocasiones, con la explicación (1f):

(1f) Identificar los diferentes sintagmas o grupos de palabras en enunciados y textos, diferenciando la palabra nuclear del resto de palabras que lo forman y los mecanismos de conexión entre estas y el núcleo y explicando su funcionamiento en el marco de la oración simple. (Secundaria-2. ${ }^{\circ}$ Castilla La Mancha-L1).

(1g) Identificar los conectores textuales y los principales mecanismos de referencia interna (tanto gramaticales como léxicos) presentes en los textos reconociendo la función que realizan en la organización del contenido del discurso. (Secundaria-2. ${ }^{\circ}$ Castilla La Mancha-L1).

En los datos de contraste de la figura 4, cabe detenerse en el caso de L1euskera, que comprende tres documentos (País Vaseo ${ }^{8}$, Navarra-modelo A ${ }^{9}$ y Navarra-modelo D). En primaria, la categoría «Reconocer» ofrece un porcentaje del $43,07 \%$, mientras que en secundaria este porcentaje desciende hasta el 16,42\%.

En primaria, en el currículo del País Vasco, junto a ejemplos similares a los mostrados anteriormente, observamos otros en que la identificación va asociada al contraste de lenguas:

(1h) Identificación de los calcos y transferencias entre las diferentes lenguas. (Primaria-Primer ciclo $\left(1 .^{\circ}-2 .^{\circ}-3{ }^{\circ}\right)$. País Vasco-L1-euskera-castellano).

En el caso de los currículos de euskera de Navarra, el documento del Modelo A individualiza la relación entre identificación e ítems gramaticales, como en (1i, 1j,1k):

(1i) Conoce los casos más usuales: sociativo, inesivo, ablativo, adlativo, destinativo e instrumental. (Primaria: $3 .^{\circ}$ Navarra. L1-euskera, modelo A).

(1j) Identifica el grado (repetición del adjetivo, adverbios gehiago y gutxiago). (Primaria: 3. ${ }^{\circ}$ Navarra. L1-euskera, modelo A).

(1k) Identifica los aspectos verbales (burutua y etorkizunekoa). (Primaria: 3. ${ }^{\circ}$ Navarra. L1-euskera, modelo A).

En cambio, el currículo de euskera de Navarra-modelo D, presenta la identificación en relación con ítems ǵramaticales aǵrupados, como en (11):

(11) Identificar y utilizar correctamente los sustantivos (comunes y propios), demostrativos, cuantificadores, pronombres personales y los adjetivos calificativos. (Primaria: 2. ${ }^{\circ}$ Navarra. L1-euskera, modelo D).

8 En este caso, el currículo de L1 es común para euskera y castellano.

9 Modelo A: enseñanza en castellano, con el euskera como asignatura. Modelo D: enseñanza en euskera, con el castellano como asignatura (https://www.educacion.navarra.es/web/dpto/modelos-linguisticos). 
En el currículo de secundaria, en el caso del País Vasco especialmente, también adquiere especial importancia el contraste de lenguas. Pero, frente al predominio de la identificación en primaria, en secundaria se hace hincapié en la reflexión a partir del análisis, lo que se refleja en la categoría de Evaluar (32,90\%), que comentaremos más adelante.

La categoría «Comprender» (CONOC-CÓMO-COMPREN), según Bloom-Anderson y Krathwohl (2001), es la habilidad de construir significado a partir de material educativo, como la lectura o las explicaciones del docente. En los currículos, podemos rastrearla mediante indicadores como captar, comprender, definir, interpretar o resumir. Su delimitación no siempre resulta fácil, dadas las concomitancias, en ocasiones, con la identificación (que se puede entender como un nivel básico de comprensión) y, especialmente, con la categoría "Analizar»(CONOC-CÓMO-ANALIZ), que implica descomponer el conocimiento en sus partes y pensar en cómo estas se relacionan con su estructura global. Consideramos operaciones de análisis asociar, clasificar, comparar, deducir, distinguir, diferenciar, inferir u observar (con el sentido de «examinar atentamente»). Por ello, interpretamos los datos de ambas cateǵorías en correlación (fig. 6).

\begin{tabular}{|lcc|}
\hline \multicolumn{1}{|c}{ Documentos curriculares } & $\begin{array}{c}\text { Categorías: } \\
\text { Comprender - Analizar } \\
\text { primaria }\end{array}$ & $\begin{array}{c}\text { Categorías: } \\
\text { Comprender - Analizar } \\
\text { secundaria }\end{array}$ \\
\hline L1-Castellano & $5,20 \%-13,84 \%$ & $0,46 \%-20,54 \%$ \\
\hline L1-Catalán & $6,00 \%-17,52 \%$ & $1,40 \%-17,40 \%$ \\
\hline L1-Euskera & $6,22 \%-20,11 \%$ & $1,33 \%-8,23 \%$ \\
\hline L1-Gallego, aragonés, asturiano & $5,57 \%-14 \%$ & $2,47 \%-18 \%$
\end{tabular}

Figura 6. Categorías: Comprender y Analizar. Presencia en currículos de L1, primaria-secundaria (elaboración propia).

La categoría «Comprender», separada del análisis, tiene poca presencia en los currículos, tanto de primaria como de secundaria. Dado que se trata de formación de conceptos, como los ítems gramaticales en este caso, no parece que ambas categorías puedan ir separadas con facilidad. De acuerdo con Barth (2004), la comparación (análisis), tras la percepción significativa, es necesaria para que se produzca el proceso de abstracción y se pueda ir asentando un concepto.

Las concomitancias de estas categorías con la de «Reconocer» son muy frecuentes en los documentos curriculares, como se puede observar en los ejemplos 
siguientes: 2a-2d. En 2a (primaria), la identificación no se puede realizar sin el análisis de la función que la palabra realiza y en $2 \mathrm{~b}-2 \mathrm{~d}$ (secundaria), la identificación se une al análisis de las relaciones morfosintácticas que contraen los elementos que conforman cada grupo de palabras:

(2a) Identifica en un texto algunas palabras por su función en la lengua: presentar al nombre, expresar características del nombre, expresar acciones o estados, enlazar o relacionar palabras $\mathrm{u}$ oraciones. (Primaria: $4 .^{\circ}$ Cantabria. L1).

(2b) Identificación de los grupos de palabras y sus tipos, así como de las relaciones morfosintácticas entre los elementos que conforman el sintagma nominal, el adjetival, el preposicional y el adverbial. (Secundaria: $2{ }^{\circ} \mathrm{C}$. Valenciana. L1).

(2c) Identificación y análisis morfosintáctico del sujeto. El sujeto omitido y el sujeto múltiple. Ausencia del sujeto. (Secundaria: $2{ }^{\circ}$ C. Valenciana. L1).

(2d) Clasificación de la oración simple según la naturaleza del predicado (oraciones atributivas y predicativas —impersonales-) y según la actitud del hablante (modalidades oracionales). (Secundaria: $2{ }^{\circ} \mathrm{C}$. Valenciana. L1).

La categoría «Aplicar» (CONOC-CÓMO-APLIC), según Bloom-Anderson y Krathwohl (2001), implica la aplicación de un proceso aprendido, ya sea en una situación familiar o en una nueva. En los currículos de L1, esta categoría recoge, de forma mayoritaria, la aplicación de normas ortográficas, vinculadas a la corrección en el uso de la lengua. En menor medida, aparecen ítems que implican manipulación de unidades como cambiar (de orden), completar, emplear, insertar, ordenar, recomponer, segmentar, suprimir o transformar, cuyos efectos pueden propiciar la reflexión sobre el funcionamiento la lengua, motivar la reflexión metalinguuística, como señalan Chartrand (2003) y Zayas (2011b).

En la figura 7, recogemos el contraste de porcentajes de esta categoría en primaria y secundaria.

\begin{tabular}{|lc|}
\hline Documentos curriculares & $\begin{array}{c}\text { Categoría: Aplicar } \\
\text { primaria - secundaria }\end{array}$ \\
\hline L1-Castellano & $27,50 \%-20,48 \%$ \\
\hline L1-Catalán & $23,92 \%-21,60 \%$ \\
\hline L1-Euskera & $10,98 \%-17,11 \%$ \\
\hline L1-Gallego, aragonés, asturiano & $35,81 \%-27,33 \%$
\end{tabular}

Figura 7. Categoría: Aplicar. Presencia en currículos de L1, primaria-secundaria (elaboración propia). 
Los ítems que se refieren a la manipulación de unidades, menos presentes que los de corrección, como ya hemos señalado, aluden a operaciones mayoritariamente sintácticas como la inserción, la transformación, la sustitución o la ampliación. En todos los casos, se señala que estas operaciones han de estar al servicio de la mejora del uso (comprensión o expresión):

(3a) Inserción y coordinación de oraciones como instrumento en la mejora de la composición escrita. (Primaria: $3{ }^{\circ}$ Galicia. L1-gallego).

(3b) Establecer concordancias de género y número con palabras cuyo significado cambia con el género (el orden, la orden) y con las que son comunes o ambiguas en cuanto al género (el/la oficinista, el/la mar, el/la azúcar). (Primaria: 4. ${ }^{\circ}$ Madrid. L1).

(3c) Identificar el papel semántico del sujeto y transformar oraciones activas en pasivas, y viceversa, para mejorar la comprensión y la producción de textos. (Primaria: 6. ${ }^{\circ}$ Galicia. L1-gallego).

(3d) Reconocer y usar los mecanismos de sustitución pronominal y adverbial para evitar repeticiones en el discurso oral y escrito. (Primaria: $6 .^{\circ}$ Asturias. L1-asturiano).

(3e) Transforma y amplía oraciones simples en oraciones compuestas usando conectores y otros procedimientos de sustitución para evitar repeticiones. (Secundaria $4 .^{\circ}$ La Rioja. L1).

(3f) Reconoce y utiliza la sustitución léxica como un procedimiento de cohesión textual. (Secundaria-4. ${ }^{\circ}$ Baleares. L1).

Las dos últimas categorías («Evaluar»y «Crear») son las que requieren operaciones cognnitivas más complejas. La evaluación requiere comprobación y crítica, mientras que la creación (categoría añadida por Anderson y Krathwohl, 2001 a la propuesta inicial de Bloom) implica reunir cosas y hacer algo nuevo. En nuestro caso, estas dos categorías nos sitúan ante el control del uso, no ante el análisis únicamente. Por ello, en «Evaluar» tiene especial importancia la revisión y en «Crear», la planificación, ya que en ambos casos ha de estar presente la reflexión sobre la lenǵua. Estos ítems aparecen, en algunos casos, en relación con contenidos gramaticales del bloque de Conocimiento de la lengua, pero, sobre todo, en relación con los contenidos de escritura (Comunicación escrita: escribir). Con frecuencia, asimismo, planificación y revisión van asociadas, como fases del proceso de escritura.

La categoría «Evaluar» (CONOC-CÓMO-EVAL) es la última categoría de análisis y requiere de un componente de valoración, que se refleja en indicadores como comprobar (la ortografía), detectar (errores), evaluar, explicar, extraer regularidades, jusgar, proponer (soluciones), reconocer (los errores), resolver (dudas), revisar, valorar. 
Los resultados obtenidos se recogen en la figura 8.

\begin{tabular}{|lc|}
\hline Documentos curriculares & $\begin{array}{c}\text { Categoría: Evaluar } \\
\text { primaria - secundaria }\end{array}$ \\
\hline L1-Castellano & $10 \%-19,80 \%$ \\
\hline L1-Catalán & $17,10 \%-16,42 \%$ \\
\hline L1-Euskera & $6,22 \%-32,90 \%$ \\
\hline L1-Gallego, aragonés, asturiano & $12,54 \%-16,83 \%$
\end{tabular}

Figura 8. Categoría: Evaluar. Presencia en currículos de L1, primaria-secundaria (elaboración propia).

En esta categoría, se observa la importancia concedida a los aspectos normativos (comprobar ortografía, detectar o reconocer errores), pero, junto a ellos, también encontramos la explicación, como forma de evaluación de contenidos gramaticales $(4 a-4 b)$ :

(4a) Reconocer la concordancia de los elementos constitutivos de la oración para construir oraciones simples adecuadas al nivel, detectando errores y resolviendo sus dudas mediante un proceso guiado de reflexión individual y colectiva. (Primaria-4. ${ }^{\circ}$ Comunidad Valenciana. L1).

(4b) Observar, reconocer y explicar los usos de los grupos nominales, adjetivales, verbales, preposicionales y adverbiales dentro del marco de la oración simple. (Secundaria-3. ${ }^{\circ}$ Extremadura. L1).

Y especialmente de la revisión (4c-4ǵ), como operación de escritura que no es posible sin reflexión metalingüüstica (Rodríguez-Gonzalo, 2014), que aparece de manera constante desde los primeros cursos en relación con los contenidos de comunicación escrita (lectura-escritura), no con los de conocimiento de la lengua.

(4c) Revisar el texto que se ha escrito y mejorar su coherencia y cohesión, el léxico y la puntuación. (Primaria-primer ciclo $\left(1 .^{\circ}\right.$ y $\left.2 .^{\circ}\right)$. Cataluña. L1-catalán y castellano).

(4d) Realizar, mediante procedimientos guiados, el proceso de revisión de textos escritos, detectando errores, de forma reflexiva y dialogada, con ayuda de sus compañeros para mejorar el producto final y presentarlo cuidando los aspectos formales y respetando las normas de corrección gramatical y ortográfica del nivel educativo. (Primaria-3. ${ }^{\circ}$ C. Valenciana. L1).

(4e) Revisa el texto en varias fases para aclarar problemas con el contenido (ideas y estructura) o la forma (puntuación, ortografía, gramática y presenta- 
ción) evaluando su propia producción escrita o la de sus compañeros. (Secundaria-1. ${ }^{\circ}$ Canarias. L1).

(4f) Emplear la reflexión gramatical en el nivel de la frase y del texto para resolver problemas de comprensión de textos escritos y multimedia, y para revisar con progresiva autonomía los textos propios y ajenos. (Secundaria-2. ${ }^{\circ} \mathrm{Ca}-$ taluña. L1-catalán y castellano. Dimensión de comprensión lectora).

(4g) Aplicar los conocimientos sobre la lengua y sus normas de uso para resolver problemas de comprensión de textos orales y escritos y para la composición y revisión progresivamente autónoma de los textos propios y ajenos, utilizando la terminología gramatical necesaria para la explicación de los diversos usos de la lengua. (Secundaria-3. ${ }^{\circ}$ Madrid. L1).

Los datos relativos a los currículos de euskera merecen un comentario por el desequilibrio de los porcentajes entre primaria y secundaria. El currículo de primaria L1, en el caso del País Vaseo, se organiza en dos ciclos (primer ciclo: cursos $1 .^{\circ}, 2 .^{\circ}$ y $3 .^{\circ}$; segundo ciclo: cursos $\left.4 .^{\circ}, 5 .^{\circ}, 6 .^{\circ}\right)$, y en ellos los ítems relacionados con la reflexión sobre el sistema y con la revisión se agrupan y aparecen una sola vez por ciclo (4h-4i), lo que no ocurre en secundaria, en que se desǵlosan por cursos y, además, se acentúa la presencia de los ítems relacionados con la reflexión sobre los contenidos del sistema $(4 \mathrm{j}-4 \mathrm{k})$.

(4h) Reflexionar sobre el sistema y las normas de uso de la lengua, mediante la comparación y transformación de textos, enunciados y palabras; y utilizar estos conocimientos para solucionar problemas de comprensión y en los procesos de textualización y revisión dirigida de los textos, afianzando, asimismo, el uso del sistema de escritura. (Primaria-primer ciclo $\left(1 .^{\circ}, 2 .^{\circ}, 3 .^{\circ}\right)$. País Vasco. L1 euskera y castellano).

(4i) Participa, de modo guiado, en las tareas de revisión y mejora de las producciones tanto propias como ajenas y valora las aportaciones ajenas. (Primaria-segundo ciclo $\left(4 .^{\circ}, 5 .^{\circ}, 6^{\circ}\right)$. País Vasco. L1 euskera y castellano).

(4j) Extrae regularidades sobre el sistema lingüuístico completando, comparando y transformando textos o enunciados según criterios dados. (Secundaria1. ${ }^{\circ}$ País Vasco: L1 euskera y castellano).

(4k) Reflexionar sobre el sistema y las normas de uso de la lengua, mediante la comparación y transformación de textos, enunciados y palabras, y utilizar estos conocimientos para solucionar, de manera guiada, problemas de comprensión y para mejorar los procesos de textualización y revisión dirigida de los textos. (Secundaria-2. ${ }^{\circ}$ País Vasco: L1 euskera y castellano).

Por último, en la categoría «Crear» (CONOC-CÓMO-CREAR), se han rastreado únicamente los ítems en que se alude al proceso de elaboración de textos en la fase de planificación y generación de ideas, con indicadores como idear, generar ideas o planificar, que suponen reflexión sobre los recursos lingüuísticos impli- 
cados en la forma de expresar las ideas. La elaboración de textos en su conjunto se ha considerado en las categorías de Uso de la lenǵua (v. figura 2), que no son objeto de análisis en este artículo, de ahí que los porcentajes de aparición en este caso sean reducidos.

\begin{tabular}{|lc|}
\hline Documentos curriculares & $\begin{array}{c}\text { Categoría: Crear } \\
\text { primaria - secundaria }\end{array}$ \\
\hline L1-Castellano & $9,73 \%-4,52 \%$ \\
\hline L1-Catalán & $10,52 \%-10,33 \%$ \\
\hline L1-Euskera & $13,40 \%-21,01 \%$ \\
\hline L1-Gallego, aragonés, asturiano & $8,82 \%-3,02 \%$
\end{tabular}

Figura 9. Categoría: Crear. Presencia en currículos de L1, primaria-secundaria (elaboración propia).

Los ítems relacionados con esta categoría quedan ejemplificados en 5a-5d:

(5a) Planificar y escribir, de forma reflexiva y dialogada, con la supervisión de un adulto y la colaboración de sus compañeros, textos de los géneros más habituales del nivel educativo, redactando el borrador, con una estructura coherente y un vocabulario apropiado y utilizando los recursos lingüústicos con creatividad y sentido estético. (Primaria-5. ${ }^{\circ}$ C. Valenciana. L1).

(5b) Organiza y planifica el discurso adecuándose a la situación de comunicación y a las diferentes necesidades comunicativas (narrar, describir, informarse, dialogar) utilizando los recursos lingüústicos pertinentes. (Primaria-6. ${ }^{\circ}$ Aragón. L1 castellano).

(5c) Escribe textos usando el registro adecuado, organizando las ideas con claridad, enlazando enunciados en secuencias lineales cohesionadas y respetando las normas gramaticales y ortográficas. (Secundaria-1. ${ }^{\circ}$ Extremadura. L1).

(5d) Planificar, producir y revisar el escrito a fin de producir textos adecuados, coherentes, cohesionados y correctos gramaticalmente. (Secundaria-1. ${ }^{\circ}$ Galicia. L1-gallego).

Esta exploración de las operaciones cognitivas nos permite establecer dos gradaciones (tanto en primaria como en secundaria) en la adquisición de los conocimientos gramaticales, que toman como punto de partida la categoría «Reconocer». La primera, formada por las operaciones de comprender, analizar y evaluar, correspondería al proceso de adquisición de los conceptos propio del aprendizaje de la abstracción (Barth, 2004). La segunda, que incluiría las operaciones de aplicar (transformar, sustituir, completar, cambiar de orden...) y crear, estaría relacionada con el control del uso. La relación entre ambas, es decir, entre 
el análisis y el control es la clave del uso reflexivo, pero, como señala la investigación didáctica (Ribas, Fontich y Guasch, 2014; Rodríguez Gonzalo, 2015; Camps y Fontich, 2020), esta relación no se produce de modo automático, sino que necesita de planteamientos metodológicos en el aula que la propicien.

\section{Conclusiones}

El análisis de las categorías relacionadas con las operaciones cognitivas que se persiguen en relación con los conocimientos gramaticales en los documentos curriculares nos permite volver sobre la diferencia entre gramática divulgativa y gramática escolar. El currículo no es una gramática escolar, en modo alguno, pero marca tendencias, a veces aspiraciones, que pueden llevar a planteamientos no deseados en el aula.

Los currículos actuales insisten obsesivamente en recalcar la vinculación del aprendizaje gramatical con la mejora del uso, para alejar la gramática de los excesos formalistas de otras épocas. Pero esta afirmación implica riesgoos (Durán, 2010). En primer lugar, porque da la falsa sensación de que conocer una unidad gramatical y mejorar el uso de la lengua es una relación que se produce de forma casi automática (reconocer y usar, identificar para mejorar la comprensión y expresión...), lo que dista mucho de ser así. La identificación solo es el primer paso de la adquisición de un concepto (Barth, 2004) y, sin la reflexión que implican el análisis, las actividades reflexivas de aplicación o las de uso controlado (planificación, revisión, reescritura), no se puede entender el aprendizaje gramatical como desarrollo de la competencia metalingüústica. La investigación didáctica muestra que para ello se necesita de diálogo durante el aprendizaje (aprender a hablar, a razonar sobre gramática) y de acompañamiento en el aula, de prácticas reflexivas desde los primeros años de escolaridad (Camps, 2006; Fontich, 2006; Camps, 2017; Myhill, Watson y Newman, 2020), lo que también es un reto para la formación de docentes, tanto de primaria como de secundaria.

Además, en los currículos de lenǵuas primeras, especialmente en los de territorios con más de una lengua propia, se aboga por el contraste de lenguas en las distintas operaciones analizadas (en la identificación, en el análisis, en la evaluación). También para este contraste y para fomentar la transferencia entre lenguas que implica la gramática interlingüuística se necesita un planteamiento metodológico en el aula donde la reflexión se produzca como resultado de la interacción (Ruiz Bikandi, 2008, Guasch, 2011).

En segundo lugar, el efecto no deseado que ha provocado la aparente facilidad de la relación entre gramática y uso ha sido el derivar el aprendizaje gramatical únicamente hacia los aspectos normativos, que, aun siendo necesarios, empobrecen este aprendizaje y lo alejan de la reflexión. La ambigüiedad de la expresión 
«mejorar el uso» se observa en las operaciones de aplicación (aplicar normas, detectar errores...). Si solo remitimos el aprendizaje goramatical al dominio de normas, excluimos la reflexión sobre la variación (en la propia lengua y entre lenguas), sobre los efectos de significado de las distintas formas de decir y sobre la toma de decisiones de cada hablante acerca del uso consciente de los recursos que las lenguas nos ofrecen. El conocimiento gramatical nos ayuda en esta toma de decisiones (Kolln y Gray, 2014).

Los currículos vigentes muestran que la gramática escolar ha de integrar el conocimiento del sistema y de sus normas, así como las variaciones inherentes a los distintos usos en las distintas lenguas curriculares. Es difícil no estar de acuerdo con ello, aunque se discrepe en la selección de contenidos, más o menos desǵlosada o repetitiva en los distintos cursos. Pero es equívoco pensar que un aprendizaje reflexivo se consigue sin planteamientos metodológicos que lo hagan posible y que permitan a los docentes abordar la enseñanza gramatical, sin exigirles que se conviertan en expertos lingüiistas. Eliminar la oposición entre conocimiento y conducta, como plantean Bosque y Gallego (2016), no es tarea fácil y requiere de docentes formados (tanto en gramática como en didáctica) para hacerlo posible. Ser conscientes de estos riesǵos nos permite abordar el reto de una gramática escolar interlingüuística, a partir del camino ya recorrido por la investigación didáctica. Cabe esperar, asimismo, que los nuevos planteamientos curriculares consideren la importancia del desarrollo de la competencia metalingüística en la formación plurilingüe del alumnado.

\section{Referencias}

Anderson, L. W., y Krathwohl, D. R. (Ed.). (2001). A taxonomy for learning, teaching, and assessing: A revision of Bloom's Taxonomy of Educational Objectives. Longman.

Barth, B.-M. (2004). L'apprentissage de l'abstraction. Retz.

Bosque, I., y Gallego, Á. J. (2016). La aplicación de la gramática en el aula. Recursos didácticos clásicos y modernos para la enseñanza de la gramática. Revista de Lingüística Teórica y Aplicada 54(2), 63-83. https://scielo.conicyt.cl/pdf/rla/ v54n2/art_04.pdf

Bosque, I., y Gallego, Á.J. (2018). La gramática en la Enseñanza Media. Competencias oficiales y competencias necesarias. ReGrOC, 1/1, 141-201. https://doi. org $/ 10.5565 /$ rev/regroc. 20

Bowen, G. A. (2009). Document Analysis as a Qualitative Research Method. Qualitative Research Journal, 9,2, 27-40. https://doi.org/10.3316/QRJ0902027

Cambra, M. (2013). Investigar la interacción en las clases de lenguas. Cultura y Educación, 25(4), 429-440. https://doi.org/10.1174/113564013808906807 
Camps, A. (2000). Aprendre gramática. En A. Camps y M. Ferrer (Eds.). Gramàtica a l'aula (pp. 101-118). Graó.

Camps, A. (2006). Secuencias didácticas para aprender gramática. En A. Camps y F. Zayas (Coords.). Secuencias didácticas para aprender gramática (pp. 31-36). Graó.

Camps, A. (2014). Hacia una renovación de la enseñanza de la gramática. Lenguaje y Textos, 40, 7-18.

Camps, A. (2017). Reflexiones sobre la enseñanza y el aprendizaje de la gramática. En A. Camps y T. Ribas (Coords.). El verbo y su enseñanza (pp. 19-31). Octaedro.

Camps, A., y Fontich, X. (Eds.). (2020). Research and teaching at the intersection. Navigating the territory of grammar and writing in the context of metalinguistic activity. Peter Lang. https://doi.org/10.3726/b17237

Chartrand, S. G. (2003). Sept chantiers pour travailler la grammaire en classe. Québec français, 129, 73-76. https://id.erudit.org/iderudit/55757ac

Costa, A. L. (2020). Grammar teaching 91-19: an analysis of the portuguese curricula. L1-Educational Studies in Language and Literature, 20, 1-31. https://doi. org/10.17239/L1ESLL2020.20.03.03

Cuenca, M. J. (2010). Gramática del texto. Arco-Libros.

Durán, C. (2010). Les competències i l'ensenyament de la gramática. Articles de Didàctica de la Llengua $i$ la Literatura, 51, 36-50.

Ellis, R. (2016). Focus on form: A critical review. Language Teaching Research, 20(3), 405-428. https://doi.org/10.1177/1362168816628627

Ellis, V., Edwards, A., y Smagorinsky, P. (Ed.). (2010). Cultural-historical perspectives on teacher education and development: learning teaching. Routledge.

Fontich, X. (2006). Hablar y escribir para aprender gramática. ICE-Horsori.

Fontich, X., y Camps, A. (2014). Towards a rational for research into grammar teaching in schools. Research papers in education, 29(5), 598-625. https://doi.org/10.10 $80 / 02671522.2013 .813579$

Guasch. O. (2011). Plurilingüisme i formació lingüuística dels escolars. En O. Guasch (Coord.). El tractament integrat de les llengües (pp. 13-28). Graó.

Jover, G. (2008). ¿Qué currículo? Currículo y práctica docente. Textos de Didáctica de la Lengua y la Literatura, 48, 49-60.

Kolln, M., y Gray, L. (2014). Rhetorical Grammar. Grammatical Choices, Rhetorical Effects. Pearson.

Landero, L. (1999). El gramático a palos. El País, 14-XII-1999.

Locke, T. (Ed.). (2010). Beyond the Grammar Wars. A Resource for Teachers and Students on Developing Language Knowledge in the English/Literacy. Routledge.

Ministerio de Educación y Formación Profesional. (2020). El currículo a debate. Un currículo para una sociedad que avanza. https://curriculo.educacion.es/wpcontent/uploads/2021/03/Relatoria_Foro_Virtual_Curriculo_a_Debate.pdf

Myhill, D., Watson, A., y Newman, R. (2020). Thinking differently about grammar and metalinguistic understanding in writing. Bellaterra Journal of Teaching \& Learning Language \& Literature, 13(2), e870. https://doi.org/10.5565/rev/jtl3.870 
Nadeau, M., y Fisher, C. (2011). Les connaissances implicites et explicites en grammaire: Quelle importance pour l'enseignement? Quelles conséquences ? Bellaterra Journal of Teaching \& Learning Language \& Literature 4(4), 1-31. https://doi. org $/ 10.5565 / \mathrm{rev} / \mathrm{jtl} 3.446$

Nassaji, H. (2017). Com integrar un focus en la gramàtica a les classes comunicatives de llengua. Caplletra, 63, 165-188. https://doi.org/10.7203/caplletra.63.10398

Ribas, T., Fontich, X., y Guasch, O. (Eds.). (2014). Grammar at school: Research on Metalinguistic activity in language education. Peter Lang. https://doi. org/10.3726/978-3-0352-6490-6

Rodríguez Gonzalo, C. (2012). La enseñanza de la gramática: las relaciones entre la reflexión y el uso lingüústico. Revista Iberoamericana de Educación, 59, 87-118. https://doi.org/10.35362/rie590458

Rodríguez Gonzalo, C. (2014). Enseñanza de la gramática y escritura: el papel de la revisión. Lenguaje y textos, 40, 19-31.

Rodríguez Gonzalo, C. (2015). La intervención didáctica en el aula sobre gramática y escritura. El saber gramatical y la revisión de textos en el uso de los tiempos verbales del pasado con alumnos de Secundaria. Cultura y Educación, 27(4), 879-898. https://doi.org/10.1080/11356405.2015.1089388

Rodríguez-Gonzalo, C., y Durán, C. (2019) Relaciones entre conceptualización y uso en el aprendizaje de la gramática. Dos investigaciones de aula sobre el verbo. En A. Leal, F. Oliveira, F. Silva, I. M. Duarte, J. Veloso, P. Silvano y S. Valente (Orgs.). A linguística na formação do professor: das teorias às práticas (pp. 181-206). Universidade do Porto. https://doi.org/10.21747978-989-8969-20-0linga11.pdf

Ruiz Bikandi, U. (2008). La reflexión metalingüuística desde varias lenguas. Textos de Didáctica de la Lengua y la Literatura, 47, 33-45.

Ruiz Bikandi, U. (2010). El conocimiento sobre la lengua en el Decreto de Enseñanzas Mínimas de 2007. Una revisión crítica. En T. Ribas (Coord.). Libros de texto y enseñanza de la gramática (pp. 33-54). Graó.

Woods, D. (1996). Teacher cognition in language teaching: Beliefs, decision-making and classroom practice. Cambridge University Press.

Zayas, F. (2011a). El lugar de la gramática en la enseñanza de la lengua. En U. Ruiz Bikandi (Coord.). Lengua castellana y Literatura: innovación, investigación y buenas prácticas (pp. 91-106). Graó.

Zayas, F. (2011b). Hacia una tipología de actividades gramaticales. Darle a la lengua (no disponible). 
